\title{
A Descriptive Epidemiological Study of Head and Neck cancers at a Major Referral Center in Southern Africa
}

\author{
Komeela Naidoo ${ }^{1}$, Hannah Simonds ${ }^{1}$, Abdul-Kader Ebrahim ${ }^{1}$, Leon Janse vAn Rensburg ${ }^{1}$, \\ Marc Merven ${ }^{1}$, Johan Opperman ${ }^{2}$, and Amir Afrogheh ${ }^{2}$ \\ ${ }^{1}$ University of Stellenbosch \\ ${ }^{2}$ University of Western Cape Faculty of Dentistry
}

May 11, 2020

\begin{abstract}
Background The aim was to describe the profile of Head and Neck Cancers (HNC) at a referral centre in South Africa. Methods Records from January 2015 to December 2017 were reviewed. Variables analysed were site, histologic type, age, sex, HIV status, p16 status, treatment intent/ modality, and the Eastern Cooperative Oncology group performance (ECOG). Results Of 854 patients, $71 \%$ were male. Median age was 58 . Smoking was a risk factor in $86.3 \%(\mathrm{n}=737)$ and alcohol in $74.2 \%$ $(\mathrm{n}=634) .53 .86 \%(\mathrm{n}=460)$ and $27.17 \%(\mathrm{n}=232)$ had ECOG scores of 1 and 2 respectively. 9\% ( $\mathrm{n}=56)$ were HIV positive. 167 had oropharyngeal primaries, and $9.58 \%(\mathrm{n}=16)$ were p16-positive. $53.87 \%(\mathrm{n}=466)$ had locally advanced disease, and $47.42 \%$ $(\mathrm{n}=405)$ received palliative treatment. Conclusion At this centre, HNC affects older adult males of lower socioeconomic status, who often smoke and drink and present with locally advanced disease, requiring palliative oncologic care. HPV and HIV play a minor role.
\end{abstract}

\section{Introduction}

Head and Neck Cancers (HNC) constitute a major public health concern worldwide. The worldwide incidence is more than 550000 cases per yearwith approximately 350000 deaths per annum ${ }^{(1)}$. In 2017, about 63030 new cases of oral cavity, pharyngeal and laryngeal cancers were reported to occur in the USA (about $3.7 \%$ of all new cases), with an estimated 13360 deaths from HNC during the same time period ${ }^{(2)}$.

The incidence of HNC is increasing sharply in developing countries. ${ }^{(1)} \mathrm{HNC}$ ranks third in Africa with a combined Age Standardized Incidence Ratio (ASIR) of $7.8^{(3)}$ Looking at the Sub-Saharan region, HNC ranks fourth with an estimated incidence of 27593 per 100000 and a cumulative risk of $0.66{ }^{(1,3)}$. An increasing trend in the incidence of HNC in South Africa has been reported over the years from 1992 to 2001, and it was observed that mixed ancestry South Africans had the highest incidence amongst all ethnicities ${ }^{(4)}$.

There are numerous, well-established risk factors. These include tobacco, alcohol, high-risk human papilloma virus (HR-HPV) exposure, Epstein Barr Virus (EBV) infection, trauma, poor oral health, dietary deficiencies and betel leaf chewing.

The incidence of HNC increases with age, and most patients are between 50 and 70 years of age. ${ }^{(5)}$. The most common histologic type is squamous cell carcinoma, which accounts for approximately $90 \%$ of all HNC. Over $50 \%$ of $\mathrm{HNC}$ occur in men ${ }^{(5)}$. In particular, males are more likely to have HPV-positive cancers ${ }^{(5)}$.

Predictors of overall survival include older age, higher tumour stage, high alcohol consumption and HPV status ${ }^{(6)}$. Survival decreases with increasing stage of the disease. The 5 -year overall survival is $83 \%$ for localised disease, $63 \%$ for regional disease and $38 \%$ for distant disease ${ }^{(2)}$. 
HPV-positive cancers tend to have a better outcome in comparison to HPV-negative HNC. Limited data onthe epidemiological trends of HNC in Southern Africa exist. The aim of the current study was to evaluate and describe patient demographics, risk factors, tumours characteristics, prognostic factors, disease stage, treatment intent/modality, at a major referral hospital (Blinded for review) in Southern Africa. This information may help to reduce the burden of HNC in this region, through establishing sustainable research and education networks within Africa, in collaboration with the more developed world.

\section{Methods and materials}

This retrospective cross-sectional study was conducted at [Blinded for review]. Records of all patients with HNC (cancers of the lip, oral cavity, oropharynx, nasopharynx, hypopharynx and larynx) between 1 January 2015 and 31 December 2017 (3-year period) were reviewed. Variables analysed were age, sex, HIV status, p16 status (for oropharyngeal cancers), the Eastern Cooperative Oncology group (ECOG) performance status and socio-economic status.

The uniform patient fee schedule (UPFS) was used as a surrogate for socioeconomic status. This allocated category depends on the individual's/household's income per annum (in South African Rands/Euros) (See table 1). Tumours were classified according to anatomical site, and histologic type.Staging was done according to the American Joint Committee on Cancer (AJCC) TNM $7^{\text {th }}$ edition.

Treatment intent was described as radical or palliative. Radical treatment modalities consisted of surgery, chemotherapy or radiation therapy alone or in combination. Palliative treatment modalities consisted of chemotherapy, radiotherapy and/or best supportive care. Surgery is seldom used as a treatment modality in patients with palliative intent.

Data was extracted from routine patient files, and entered into a customised data collection sheet. Data from data collection sheets was entered into Microsoft Excel 2013. Statistical analysis was performed using Microsoft Excel.

\section{Results}

Data was collected from 854 patients, seen between 2015 and 2017, with 865 reported tumours. Eleven patients had two separate primaries at the time of diagnosis. There were $603(71 \%)$ males and $251(29 \%)$ females. The male to female ratio was 2.4:1. The age range was 10-89 years (median age 58 years). Table 1 , shows the distribution of patients for each age group.

Smoking was a risk factor in $737(86.3 \%)$, alcohol in $634(74.2 \%)$ with smoking and alcohol in $620(72.6 \%)$ of patients. Fifty-six (9\%) were HIV positive, $721(84 \%)$ were negative and $77(7 \%)$ had unknown HIV status.

The ECOG performance status was recorded as a score of 0 to 5 . A score of zero indicates a fully active patient, 1 restricted strenuous activity but able to carry out light duties, 2 is capable of self-care but rests for up to $50 \%$ of the day, 3 is capable of limited self-care and rests for The scores were 1 in 460 (53.86\%), 2 in $232(27.17 \%), 3$ in 128 (14.89\%), 4 in $27(3.16 \%)$ and unknown in two $(0.23 \%)$ patients.

Table 2 and 3 shows uniform patient fees schedule (UPFS), which is a surrogate for the socio-economic status of patients. Majority of thepatients had no reported income.

The most common site was the oral cavity $(\mathrm{n}=320)$ followed by the larynx $(\mathrm{n}=188)$ and the oropharynx $(\mathrm{n}=167)$. The most common sub-site was the anterior tongue $(\mathrm{n}=137)$ followed by the supraglottic larynx $(\mathrm{n}=98)$. Appendix 1 shows the distribution of disease according to anatomical site.

The most predominate histological type was squamous cell carcinoma (SCC), which accounted for $92.6 \%$ $(\mathrm{n}=791)$ of all cases. In patients who had two synchronous primaries, both primaries were found to be SCC.

There were $20.37 \%(\mathrm{n}=174)$ grade I or well differentiated SCC, $47.90 \%(\mathrm{n}=409)$ grade II or moderately differentiated SCC, $6.79 \%(n=58)$ grade III or poorly differentiated SCC and $15017.56 \%(n=150)$ SCC with unknown histologic grade. 
Of 167 patients with oropharyngeal primaries, 9.58\% ( $\mathrm{n}=16)$ patients had p16 positive SCC (with p16 antibody showing nuclear and cytoplasmic positivity in more than $70 \%$ of the tumour cells), $46.70 \%$ ( $\mathrm{n}=78$ ) were p16 negative and the p16 status was unknown in $43.7 \%(\mathrm{n}=73)$. Three $(18.75 \%)$ of the p16 positive oropharyngeal lesions were HPV-DNA positive by Polymerase Chain Reaction (PCR).

The majority of patients presented with locally advanced disease. $466(53.87 \%)$ had stage IVA disease at presentation. Eight patients had two primaries at the time of diagnosis and both of these were stage IVA. Patients with two primaries had each primary staged separately. Distant metastases were diagnosed in 53 patients, of which 35 had lung metastases, 10 had multiple sites, two presented with bone metastases and six patients had other sites for distant disease. Table 4. shows the sub-site distribution according to the TNM stage grouping.

$449(52.58 \%)$ patients were treated with radical/curative intent and $405(47.42 \%)$ received palliative treatment. In the radical treatment group one patient had surgery followed by definitive chemoradiation and one patient only received induction chemotherapy. Four of the 11 patients with second primaries were treated with palliative intent. Table 5 . shows the distribution of various treatment modalities, according to treatment intent.

\section{Discussion}

HNCs constitute a major public health concern worldwide. There has been a significant increase in the global incidence of HNC, which is increasing sharply in developing countries ${ }^{(1)}$. Studies from South America and West Africa have shown a similar burden of disease ${ }^{(7-9)}$. Limited data onthe epidemiological trends of HNC in Southern Africa exist. The aim of the current study was to describe the profile of HNC at a major referral public hospital (TBH, Western Cape, South Africa) in Southern Africa. This information may help to develop effective education and research efforts by local and international non-governmental agencies to help reduce the mortalities and morbidities associated with HNC.

854 patients were diagnosed with HNC between 2015 and 2017, representing $12.7 \%$ of all patients seen at the Radiation Oncology Department at TBH during this period. The median age in this cohort was 58 years, with most patients being between the ages of 51 and 70 years, similar to the global age trend observed for tobacco-associated $\mathrm{HNC}^{(5)}$. Over $70 \%$ of patients were males, consistent with the existing reports that men are two to three times more likely to develop $\mathrm{HNC}^{(5)}$. The male: female ratio was $2.4: 1$; and globally this ratio varies between $2: 1$ to $4: 1$. (10)

The most predominate histologic type was SCC, which accounted for 791 (92.6\%) of all HNC cases. Smoking was a risk factor in 737 of the subjects (86.3\%), and alcohol in 634 (74.2\%). Both smoking and alcohol were co-risk factors $72.6 \%(\mathrm{n}=620)$ of the patients. Tobacco smoking and alcohol consumption are well-established major risk factors for HNC and a synergistic effect of these two factors has been consistently reported ${ }^{(11)}$.

HR-HPV is an established risk factor for the development of Oropharyngeal Squamous Cell Carcinoma (OPSCC). There is significant geographic heterogeneity in the prevalence of HPV-positive OPSCC.

Of 167 patients with oropharyngeal primaries, 9.58\% $(n=16)$ had p16-positive SCC.46.70\% ( $\mathrm{n}=78)$ were p16-negative and the p16 status was unknown in $43.7 \%(n=73)$. Only 3 of the p16 positive OPSCC were HPV-DNA positive, suggesting that p16 appears to have a low positive predictive value in our setting, and may not be used as a standalone HPV test or a surrogate marker for HR-HPV infection. Eleven of the patients with p16-positive tumours had both smoking and alcohol as risk factors, and it appears that mechanismsother than HR-HPV infection may be involved (e.g. Rb mutation) in p16 overexpression.

A large number of oropharyngeal primaries $(43.7 \%)$ had unknown HPV status, due to the fact that p16 became a routine test for oropharyngeal primaries at this hospital in recent years. Thus, we are unable to draw conclusions about the overall prevalence of HPV-positive OPSCC at TBH during the study period. However, given the very low incidence of true HPV-positive cases in the cohort of OPSCC cases with known p16 status $(3 / 94=3.2 \%)$, it may be speculated that HR-HPV exposure is possibly not a major risk factor for the development of OPSCC at this centre. 
Indeed, the increasing incidence of $\mathrm{HNC}$ in Southern Africa is mostly due to increasing smoking and drinking habits, and that preventative strategies/campaigns should primarily target tobacco and alcohol industries. Nevertheless, many countries in Africa are undergoing economic modernization, resulting in major changes in lifestyle, diet and sexual behaviour and in coming years we may observe an increasing incidence of HPVpositive OPSCC.

Fifty-six (9\%) patients were HIV positive, 721 (84\%) were HIV negative and 77 (7\%) had unknown HIV status. The HIV prevalence in the Western Cape was $12.6 \%$ in $2017^{(12)}$. The South African National HIV Prevalence, Incidence and Behaviour Survey in 2012 reported an HIV prevalence of $7.6 \%$ in individuals over 50 years of age ${ }^{(12)}$. The prevalence of HIV infection in our cohort of patients with the median age of 58 years, is similar tothe nationwide prevalence of $9 \%$ in patients over 50 years of age. It appears that HIV infection plays a minor role in the development of HNC in South Africa. The exact mechanism of HIV promoted carcinogenesis is not known ${ }^{(13)}$.

According to the 2011 South African census, low-income households are classified as those with a combined annual household income of below R19200 (\$1280). In this study, $56 \%$ of the patients fall into the low-income category. Studies have shown an increased risk of HNC in individuals of lower socioeconomic status, even after controlling for other risk factors such as smoking and alcohol consumption ${ }^{(14,15)}$. We can deduct from the latter that comprehensive HNC control programmes should also address the social inequalities and the high unemployment rate in Southern African countries.

The most common anatomical site in this study was the oral cavity $(320,37 \%)$, followed by the larynx (188, $21.73 \%)$ and the oropharynx $(167,19.31 \%)$. The most common sub-site was the anterior tongue, accounting for $15.84 \%$ (137) of the cases, followed by supraglottis $11.33 \%$ (98) and the glottis $10.40 \%$ (90), in descending order of frequency. The Surveillance, Epidemiology, and End Results [SEER] Programme reports that the commonest site for HNC is the oral tongue followed by the larynx ${ }^{(16)}$.

Field cancerization describes the presence of premalignant fields surrounding the primary tumour and has been related to the high rate of local recurrence in HNC as well as the development of synchronous primaries (17) Synchronous cancer is defined as two or more neoplasms identified simultaneously in the same patient. It is estimated that 1 to $6 \%$ of the patients diagnosed with SCC of head and neck will have a synchronous primary in the head and neck region ${ }^{(18)}$. In this study eleven patients $(1.3 \%)$ presented with a synchronous primary in the head and neck region.

According to the Surveillance, Epidemiology, and End Results (SEER) database, in the United States 42$62 \%$ of patients with HNC presented with locally advanced disease and $16-27 \%$ with distant metastases ${ }^{(2)}$. An epidemiological review of head and neck patients at a university hospital in Brazil reported that $47.8 \%$ of patients had T3/T4 disease and $31.9 \%$ had node positive disease ${ }^{(7)}$. In the current study, a significant number of patients presented with locally advanced disease $(53.87 \%, \mathrm{n}=466)$, consistent with the data from the SEER database. The incidence of distant metastases was $6.21 \%$, which is lower than that of patients presenting in the United States.

The treatment intent and modality offered to patients depends on multiple factors, i.e. stage at presentation, performance status, co-morbidities, social circumstances as well as patient wishes. In our patient group, 449 $(52.6 \%)$ patients had treatment with radical intent and $405(47.4 \%)$ with palliative intent. The majority of patients in the radical intent group had surgery (72.4\%) and approximately the same proportion of patients received radiotherapy as part of their treatment. 226 patients (50.3\%) received combined modality, including surgery, chemotherapy and radiotherapy.

Of the patients that received palliative intent, $76.5 \%$ received radiotherapy and $21.5 \%$ were not fit for any treatment and received medical palliation and best supportive care. Just less than half of the patients were for palliative intent; this could be due to the late stage at presentation. The latter could be attributed to poor socio-economic status, lack of knowledge and understanding, problems accessing health services, cultural and religious beliefs, and possible other unknown factors i.e. genetic factors in this patient population. 


\section{Conclusion}

It appears that the increasing incidence of HNC in Southern Africa is mostly due to increasing smoking and drinking habits, and that HR-HPV and HIV play a minor role in the development of HNC in this region. Nevertheless, many countries in Africa are undergoing economic modernization, resulting in major changes in lifestyle, diet and sexual behaviour and in coming years we may observe an increasing incidence of HPV-positive OPSCC. Furthermore, we have shown that SCC of the head and neck in Southern Africa is predominantly a disease of older adult males of lower socioeconomic status, who often present with locally advanced disease, requiring palliative oncologic care. This calls for an urgent adoption of HNC control programmes, that not only regulate the tobacco and alcohol industries in this region but also address the social inequalities and the high unemployment rate in this region, in the quest to reduce the mortalities and morbidities associated with HNC.

Tables

Table 1. Age distribution of head and neck cancer patients

\begin{tabular}{ll}
\hline Age group & Number of patients (\%) \\
\hline$[?] 30$ & $15(1.76)$ \\
$31-40$ & $20(2.34)$ \\
$41-50$ & $140(16.39)$ \\
$51-60$ & $331(38.76)$ \\
$61-70$ & $246(28.81)$ \\
$71-80$ & $82(9.60)$ \\
$>80$ & $20(2.34)$ \\
\hline
\end{tabular}

Table 2Table 2. Uniform patient fee schedule

Tariff category

H0- patients under the age of 18 , pensioners and the unemployed

H1

H2

H3
Household gross income per annum (01/02/200

H0- patients under the age of 18 , pensioners and the $u$ Less than R50 000(\euro3200)

More than or equal to R50000 (\euro3200) but less tha More than or equal to R100000 ( $\backslash$ euro6400)

Table 3 . Socio-economic status of head and neck cancer patients.

\begin{tabular}{ll}
\hline UPFS & Number of patients (\%) \\
\hline 0 & $339(39.70)$ \\
1 & $403(47.19)$ \\
2 & $45(5.27)$ \\
3 & $20(2.34)$ \\
Medical aid & $24(2.81)$ \\
Unknown & $23(2.69)$ \\
\hline
\end{tabular}

Table 4 . The sub-site distribution according to the TNM stage grouping. Number of patients $\mathrm{n}=854$. Number of tumours $n=865$

\section{Stage grouping Anatomical site Number of tumours (\%)}




\begin{tabular}{|c|c|c|}
\hline \multirow[t]{3}{*}{ I } & Oral cavity & $34(3.93)$ \\
\hline & Larynx & $23(2.66)$ \\
\hline & Other & $5(0.58)$ \\
\hline \multirow[t]{4}{*}{ II } & Oral cavity & $24(2.81)$ \\
\hline & Larynx & $13(1.52)$ \\
\hline & Oropharynx & $8(0.94)$ \\
\hline & Other & $8(0.94)$ \\
\hline \multirow[t]{5}{*}{ III } & Larynx & $33(3.86)$ \\
\hline & Oral cavity & $20(2.34)$ \\
\hline & Oropharynx & $18(2.10)$ \\
\hline & Nasopharynx & $8(0.94)$ \\
\hline & Other & $13(1.52)$ \\
\hline \multirow[t]{7}{*}{ IVA } & Oral cavity & $207(24.24)$ \\
\hline & Oropharynx & $97(11.36)$ \\
\hline & Larynx & $93(10.89)$ \\
\hline & Hypopharynx & $27(3.16)$ \\
\hline & Salivary gland & $14(1.64)$ \\
\hline & Sinuses & $14(1.64)$ \\
\hline & Other & $22(2.58)$ \\
\hline \multirow[t]{6}{*}{ IVB } & Oropharynx & $28(3.28)$ \\
\hline & Oral cavity & $25(2.92)$ \\
\hline & Hypopharynx & $16(1.87)$ \\
\hline & Larynx & $11(1.29)$ \\
\hline & Sinuses & $10(1.17)$ \\
\hline & Other & $22(2.58)$ \\
\hline \multirow[t]{6}{*}{ IVC } & Larynx & $14(1.64)$ \\
\hline & Oropharynx & $11(1.29)$ \\
\hline & Unknown primary & $8(0.94)$ \\
\hline & Hypopharynx & $6(0.70)$ \\
\hline & Oral cavity & $5(0.58)$ \\
\hline & Other & $9(1.05)$ \\
\hline \multirow[t]{3}{*}{ IV } & Sinuses & $3(0.35)$ \\
\hline & Base of tongue & $1(0.12)$ \\
\hline & Other & $3(0.35)$ \\
\hline \multirow[t]{3}{*}{ Unstaged } & Oral cavity & $5(0.58)$ \\
\hline & Oropharynx & $3(0.35)$ \\
\hline & Other & $4(0.47)$ \\
\hline
\end{tabular}

Table 5 . Treatment modalities

\begin{tabular}{lll}
\hline Treatment intent & Treatment modality & Number of patients (\%) \\
\hline Radical & Surgery & $325(38.06)$ \\
& Radiotherapy & $32(3.75)$ \\
& Adjuvant radiotherapy & $140(16.39)$ \\
& Adjuvant chemoradiation & $56(6.56)$ \\
& Definitive chemoradiation & $93(10.89)$ \\
Palliative & Neoadjuvant/induction chemotherapy & $31(3.63)$ \\
& Surgery & $1(0.12)$ \\
& Radiotherapy & $310(36.30)$ \\
& Chemotherapy & $11(1.29)$
\end{tabular}




\begin{tabular}{lll}
\hline Treatment intent & Treatment modality & Number of patients (\%) \\
\hline & Chemotherapy and radiotherapy & $4(0.47)$ \\
& Best supportive care & $87(10.19)$ \\
\hline
\end{tabular}

Appendix 1 : Distribution of disease according to anatomical site

\begin{tabular}{llll}
\hline Site & Subsite & Number diagnosed & Percentage of total \\
\hline Oral cavity & & 320 & 36.99 \\
& Oral tongue & 137 & 15.84 \\
& Floor of mouth & 82 & 9.48 \\
& Lip & 33 & 3.82 \\
& Retromolar trigone & 30 & 3.47 \\
& Hard palate & 16 & 1.85 \\
& Alveolar ridge & 15 & 1.73 \\
& Buccal mucosa & 7 & 0.81 \\
Larynx & & 188 & 11.33 \\
& Supraglottis & 98 & 11.33 \\
Oropharynx & Glottis & 90 & 10.40 \\
& & 167 & 19.31 \\
& Tonsil & 86 & 9.94 \\
Hypopharynx & Base of tongue & 45 & 5.20 \\
& Soft palate and uvula & 28 & 3.24 \\
& Posterior pharyngeal wall & 8 & 0.92 \\
Sinuses & Pyriform fossa & 54 & 6.24 \\
& Posterior cricoid & 42 & 4.86 \\
Salivary glands & Lateral pharyngeal wall & 5 & 0.81 \\
& & 35 & 0.58 \\
& Maxilla & 22 & 4.05 \\
Nasopharynx & Nasal cavity and ethmoids & 13 & 2.54 \\
External auditory canal & 30 & 1.50 \\
Unknown primary & & 19 & 3.47 \\
Other & Parotid & 7 & 2.20 \\
\hline & Minor & 4 & 0.81 \\
& Submandibular & 25 & 0.46 \\
& & 24 & 2.89 \\
& & 5 & 1.97 \\
& & 2.77 \\
& & 0.58 \\
\hline
\end{tabular}

\section{References}

1. International Agency for Research on Cancer. GLOBOCAN 2012 v1.0, Cancer Incidence and Mortality Worldwide. http://gco.iarc.fr/. Accessed October 09, 2017.

2. Siegel RL, Miller KD, Jemal A, et al. Cancer statistics, 2016. CA: A Cancer Journal for Clinicians. 2016;66(1): 7-30. DOI: 10.3322/caac. 21332

3. Adeola HA, Afrogheh AH, Hille JJ. The burden of head and neck cancer in Africa: the status quo and research prospects. SADJ. 2018 September; 73(8): 477-488.

4. Ayo-Yusuf OA, Lalloo R, Johnson NW. Trends and ethnic disparities in oral and oro-pharyngeal cancers in South Africa, 1992-2001. Journal of the South African Dental Association 2013;68(4):168-73. 
5. Ridge AR, Mehra R, Lango MN, Galloway T. Head and Neck Tumors. Cancer Network Home of Journal Oncology. http://www.cancernetwork.com/cancer-management/head-and-neck-tumors. Published June 2, 2016. Accessed October 1, 2017.

6. Leoncini E, Vukovic V, Cadoni G, Pastorino R, Arzani D, Bosetti C, et al. Clinical features and prognostic factors in patients with head and neck cancer: Results from a multicentric study. Cancer Epidemiology. 2015;39(3): 367-374. https://doi.org/10.1016/j.canep.2015.02.004.

7. Alvarenga L, Ruiz MT, Pavarino-Bertelli EC, Ruback MJ, Maniglia JV, Goloni-Bertollo M. Epidemiologic evaluation of head and neck patients in a university hospital of Northwestern São Paulo State. Brazilian Journal of Otorhinolaryngology. 2008; 74(1):68-73. http://dx.doi.org/10.1590/S151631802012000500007.

8. Erinoso OA, Okoturo E, NA Awolola, SS Soyemi, RT Oluwakuyide. Emerging trends in the Epidemiological Pattern of Head and Neck Cancers in Lagos, Nigeria. Annals of Medical \& Health Sciences Research. 2016 Sep-Oct; 6(5): 301-7. doi: 10.4103/amhsr.amhsr_30_16.

9. Fomete B, AgbaraR, Adebayo ET, Osunde OD, Adelola DS. An epidemiological study of 270 cases of carcinoma of the head and neck region in a Nigerian tertiary health care facility. Egyptian Journal of Ear, Nose, Throat and Allied Sciences. 2017; 18(2017): 251-5. https://doi.org/10.1016/j.ejenta.2017.11.003

10. Stenson KM, Brockstein BE, Connor RF. Epidemiology and risk factors for head and neck cancer. UpToDate. https://www.uptodate.com/contents/epidemiology-and-risk-factors-for-head-andneck-cancer. Updated 2019. Accessed September 20, 2017.

11. World Health Organization International Agency for Research on Cancer. IARC Monographs on the evaluation of carcinogenic risks to Humans. IARC Monogr Eval Carcinog Risks Hum. 2004;83:1-1438. https://www.ncbi.nlm.nih.gov/books/NBK316407. Published 2004. Accessed September 20, 2017.

12. Human Sciences Research Council. South African National HIV Prevalence, Incidence and Behaviour Survey, 2012. Human Sciences Research Council website. http://www.hsrc.ac.za/en/researchoutputs/view/6871. Published 2014. Accessed January 17,2018.

13. McLemore MS, Haigentz M, Smith RV, Nuovo GJ, Alos L, CardesaA,et al Head and Neck Squamous Cell Carcinomas in HIV-Positive Patients: A Preliminary Investigation of Viral Associations. Head Neck Pathol. 2010 Jun; 4(2): 97-105. doi: 10.1007/s12105-010-0171-9

14. Al-Dakkak I. Socioeconomic status and head and neck cancer. Evid Based Dent. 2010;11(2):57-8. doi: 10.1038/sj.ebd.6400726.

15. Johnson S, McDonald JT, Corsten MJ. Socioeconomic factors in head and neck cancer. Otolaryngol Head Neck Surg. 2008 Aug;37(4):597-601. https://www.ncbi.nlm.nih.gov/pubmed/19128600. Published 2008. Accessed September 20, 2017.

16. National Cancer Institute Surveillance, Epidemiology, and End Results Program. Cancer Stat Facts: Oral Cavity and Pharynx Cancer. National Cancer Institute Surveillance, Epidemiology, and End Results Program website. https://seer.cancer.gov/statfacts/html/oralcav.html . Updated April 5, 2018. Accessed May 7, 2018.

17. Jou A, Hess J. Epidemiology and Molecular Biology of Head and Neck Cancer. Oncol Res Treat. 2017;40:328-332. https://doi.org/10.1159/000477127.

18. Jain SK, Sikora AG, Baxi SS, Morris LG. Synchronous Cancers in Patients With Head and Neck Cancer Risks in the Era of Human Papillomavirus-Associated Oropharyngeal Cancer. Cancer. 2013 May 15;119(10):1832-7. doi: 10.1002/cncr.27988.

19. National Institute for Communicable Diseases. South African National Cancer Registry. http://www.nicd.ac.za/centres/national-cancer-registry/. Updated 2019. Accessed October 09, 2017.

20. Fitzmaurice C, Allen C, Barber RM, Barregard L, Bhutta ZA, Brenner H, et al. Global, Regional, and National Cancer Incidence, Mortality, Years of Life Lost, Years Lived with Disability, and DisabilityAdjusted Life-years for 32 Cancer Groups, 1990 to 2015: A Systematic Analysis for the Global Burden of Disease Study. JAMA Oncology 2017 3(4):524-548. doi: 10.1001/jamaoncol.2016.5688.

21. National Comprehensive Cancer Network. Head and Neck Cancers: Very Advanced Head and Neck Cancers. National Comprehensive Cancer Network website. htt- 
ps://www.nccn.org/professionals/physician_gls/pdf/head-and-neck_blocks.pdf. $\quad$ Updated 2019. Accessed September 10, 2017.

22. Western Cape Government. Tygerberg Hospital Overview. https://www.westerncape.gov.za/assets/departments/health/tygerberg_hospital_information_pamphlet_-_2016.pdf. Updated October 28, 2016. Accessed August 15, 2017.

23. Kim S. Prognostic Factors in Patients with Head and Neck Cancer.Head and Neck cancer. A Multidisciplinary Approach . 3rd ed. Philadelphia: Lippincott Williams \& Wilkins; 2009.p 87-104.

24. Lambert R, Sauvaget C, de Camargo Cancela M, Sankaranarayam, R. Epidemiology of cancer from the oral cavity and oropharynx. European Journal of Gastroenterology $\& 3$ Hepatology. 2011;23(8): 633-641. doi: 10.1097/MEG.0b013e3283484795.

25. Larizadeh M, Damghani M, Shabani M. Epidemiological Characteristics of Head and Neck Cancers in Southeast of Iran. Iranian Journal of Cancer Prevention. 2014;7(2): 80-86. https://www.ncbi.nlm.nih.gov/pmc/articles/PMC4142945/\#idm140310782150208title. Accessed September 19, 2017.

26. Marur S, Forastiere A. Head and Neck Squamous Cell Carcinoma: Update on Epidemiology, Diagnosis, and Treatment. Mayo Clinic Proceedings . 2016;91(3): 386-396. doi: 10.1016/j.mayocp.2015.12.017.

27. Pai S, Westra WH. Molecular Pathology of Head and Neck Cancer: Implications for Diagnosis, Prognosis, and Treatment. Annual Review of Pathology . 2009;4: 49-10. https://doi.org/10.1146/annurev.pathol.4.110807.092158.

28. Purgina B, Pantanowitz L, Seethala RR. A Review of Carcinomas Arising in the Head and Neck Region in HIV-Positive Patients. Pathology Research International. 2011, Article ID 469150, 12 pages. http://dx.doi.org/10.4061/2011/469150.

29. Rajapakshe RMAR, Pallegama RW, Jayasooriya, PR, Siriwardena BS, Attygalla AM, Hewapathirana $\mathrm{S}$, et al. A retrospective analysis to determine factors contributing to the survival of patients with oral squamous cell carcinoma. Cancer Epidemiology. 2015;39(3): 360-366. https://doi.org/10.1016/j.canep.2015.02.011.

30. O' Sullivan B, Hunag SH, Su J, Garden AS, Sturgis EM, Dahlstrom K, et al. Development and validation of a staging system for HPV-related oropharyngeal cancer by the International Collaboration on Oropharyngeal cancer Network for Staging (ICON-S): a multicentre cohort study. The Lancet Oncology . 2016; 17(4): 440-51. doi: 10.1016/S1470-2045(15)00560-4.

31. Gillison ML, Chaturvedi AK, Anderson WF, Fakhry C. Epidemiology of Human PapillomavirusPositive Head and Neck Squamous Cell Carcinoma. J Clin Oncol. 2015 Oct 10; 33(29): 3235-3242. doi: 10.1200/JCO.2015.61.6995. 